Mohammed A. Elmakki*, Orbett T. Alexander and Andreas Roodt

\title{
The crystal structure of trans-carbonyl-(diphenylcyclohexyl-phosphine- $\mathrm{K} P$ )iodidomethyl-(2-oxopyridin-1(2H)-olato- $\left.\mathrm{K}^{2} \mathrm{O}, \mathrm{O}^{\prime}\right)$ rhodium(III), $\mathrm{C}_{25} \mathrm{H}_{28} \mathrm{INO}_{3} \mathrm{PRh}$
}

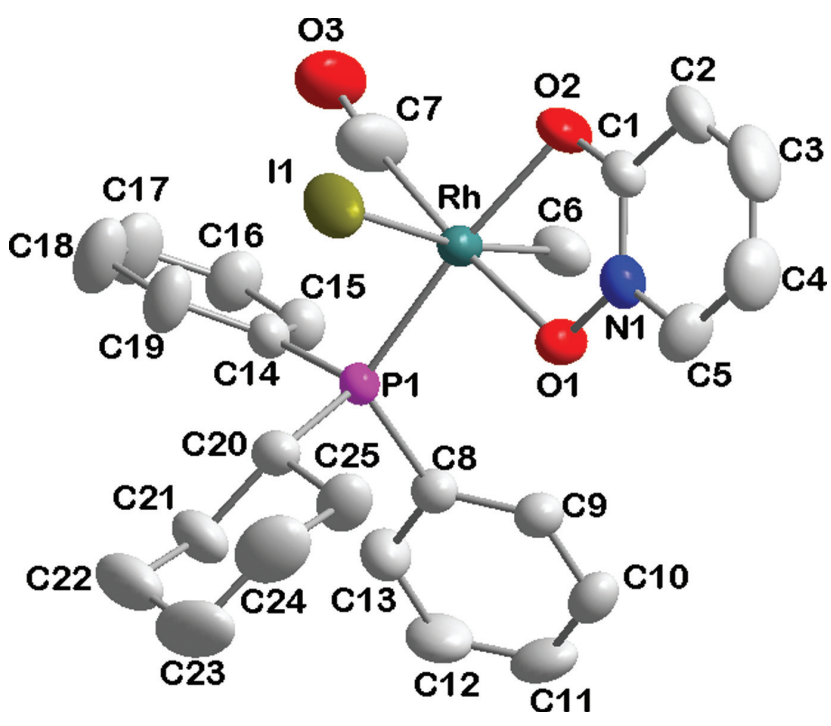

https://doi.org/10.1515/ncrs-2019-0602

Received August 20, 2019; accepted October 8, 2019; available online December 5, 2019

\begin{abstract}
$\mathrm{C}_{25} \mathrm{H}_{28} \mathrm{INO}_{3} \mathrm{PRh}$, monoclinic, $P 2_{1} / c$ (no. 14), $a=11.339(4) \AA$, $b=9.887(5) \AA, \quad c=23.069(9) \AA, \quad \beta=91.125(5)^{\circ}, \quad Z=4$, $V=2585.7(19) \AA^{3}, \quad R_{\mathrm{gt}}(F)=0.0463, \quad w R_{\mathrm{ref}}\left(F^{2}\right)=0.1093$, $T=293(2) \mathrm{K}$
\end{abstract}

\section{CCDC no.: 1958026}

Table 1 contains crystallographic data and Table 2 contains the list of the atoms including atomic coordinates and displacement parameters.

\section{Source of material}

$\left[\mathrm{Rh}\right.$ (hopo)(CO) $\left.\left(\mathrm{PPh}_{2} \mathrm{Cy}\right)\right] \quad$ (hopo $=$ 2-oxopyridin-1(2H)-olate) was synthesized according to the method described previously [5]. Rhodium(III) alkyl oxidative addition product

*Corresponding author: Mohammed A. Elmakki, Department of Chemistry, University of the Free State, Bloemfontein 9301, South Africa, e-mail: azeez77777@gmail.com. https://orcid.org/00000002-9084-3401

Orbett T. Alexander and Andreas Roodt: Department of Chemistry, University of the Free State, Bloemfontein 9301, South Africa. https://orcid.org/0000-0003-4926-8342 (0. T. Alexander)
Table 1: Data collection and handling.

\begin{tabular}{ll}
\hline Crystal: & Orange stout \\
Size: & $0.29 \times 0.15 \times 0.09 \mathrm{~mm}$ \\
Wavelength: & Mo K $\alpha$ radiation $(0.71073 \AA$ Å) \\
$\mu:$ & $1.94 \mathrm{~mm}^{-1}$ \\
Diffractometer, scan mode: & Bruker APEX-II, $\varphi$ and $\omega$ \\
$\theta_{\text {max }}$, completeness: & $28.0^{\circ}, 99 \%$ \\
$\mathrm{~N}(\mathrm{hkl})_{\text {measured }}, \mathrm{N}(\mathrm{hkl})_{\text {unique }}, R_{\text {int }}:$ & $42669,6211,0.060$ \\
Criterion for $\mathrm{I}_{\text {obs }}, \mathrm{N}(\mathrm{hkl})_{\mathrm{gt}}:$ & $\mathrm{I}_{\text {obs }}>2 \sigma\left(\mathrm{l}_{\text {obs }}\right), 4321$ \\
$N(\text { param })_{\text {refined }}:$ & 299 \\
Programs: & SHELX [1], Bruker [2], \\
& Diamond [3], Olex2 [4] \\
\hline
\end{tabular}

Table 2: Fractional atomic coordinates and isotropic or equivalent isotropic displacement parameters $\left(\AA^{2}\right)$.

\begin{tabular}{lrrrr}
\hline Atom & $\boldsymbol{x}$ & $\boldsymbol{y}$ & $\boldsymbol{z}$ & $\boldsymbol{U}_{\text {iso }}{ }^{*} \boldsymbol{U}_{\text {eq }}$ \\
\hline Rh1 & $0.39428(3)$ & $0.63865(4)$ & $0.66930(2)$ & $0.03485(11)$ \\
P1 & $0.24271(10)$ & $0.62125(12)$ & $0.60246(5)$ & $0.0307(3)$ \\
O1 & $0.3885(3)$ & $0.8453(3)$ & $0.67480(15)$ & $0.0437(8)$ \\
O2 & $0.5274(3)$ & $0.6740(4)$ & $0.73280(16)$ & $0.0510(9)$ \\
N1 & $0.4818(4)$ & $0.8902(4)$ & $0.70546(18)$ & $0.0421(10)$ \\
C1 & $0.5516(4)$ & $0.8015(5)$ & $0.7350(2)$ & $0.0361(10)$ \\
C2 & $0.6471(5)$ & $0.8537(7)$ & $0.7673(2)$ & $0.0514(14)$ \\
H2 & 0.6970 & 0.7948 & 0.7875 & $0.062^{*}$ \\
C3 & $0.6682(6)$ & $0.9894(8)$ & $0.7696(3)$ & $0.0682(19)$ \\
H3 & 0.7318 & 1.0227 & 0.7913 & $0.082^{*}$ \\
C4 & $0.5939(6)$ & $1.0776(7)$ & $0.7393(3)$ & $0.0649(17)$ \\
H4 & 0.6068 & 1.1704 & 0.7412 & $0.078^{*}$ \\
C5 & $0.5022(5)$ & $1.0274(5)$ & $0.7070(3)$ & $0.0517(14)$ \\
H5 & 0.4533 & 1.0858 & 0.6860 & $0.062^{*}$ \\
C6 & $0.2768(5)$ & $0.6207(6)$ & $0.7377(2)$ & $0.0479(13)$ \\
H6A & 0.2102 & 0.5672 & 0.7255 & $0.072^{*}$ \\
H6B & 0.2504 & 0.7090 & 0.7490 & $0.072^{*}$ \\
H6C & 0.3159 & 0.5778 & 0.7701 & $0.072^{*}$ \\
C8 & $0.1078(4)$ & $0.7094(5)$ & $0.6209(2)$ & $0.0354(10)$ \\
C9 & $0.1097(4)$ & $0.8248(5)$ & $0.6558(2)$ & $0.0398(11)$ \\
H9 & 0.1812 & 0.8568 & 0.6707 & $0.048^{*}$ \\
C10 & $0.0071(5)$ & $0.8915(5)$ & $0.6681(2)$ & $0.0479(13)$ \\
H10 & 0.0092 & 0.9669 & 0.6923 & $0.057^{*}$ \\
C11 & $-0.0999(5)$ & $0.8471(6)$ & $0.6448(3)$ & $0.0577(15)$ \\
H11 & -0.1695 & 0.8924 & 0.6531 & $0.069^{*}$ \\
C12 & $-0.1018(5)$ & $0.7348(7)$ & $0.6089(3)$ & $0.0593(16)$ \\
H12 & -0.1729 & 0.7053 & 0.5925 & $0.071^{*}$ \\
C13 & $0.0002(4)$ & $0.6668(6)$ & $0.5974(2)$ & $0.0480(13)$ \\
H13 & -0.0025 & 0.5910 & 0.5736 & $0.058^{*}$ \\
\hline (c) BY & Thiswork is licensed under the Creative Commons Attribution \\
& & & &
\end{tabular}


Table 2 (continued)

\begin{tabular}{|c|c|c|c|c|}
\hline Atom & $x$ & $y$ & $z$ & $U_{\text {iso }}{ }^{*} / U_{\text {eq }}$ \\
\hline C14 & $0.1986(4)$ & $0.4456(5)$ & $0.5930(2)$ & $0.0365(11)$ \\
\hline C15 & $0.1191(5)$ & $0.3853(5)$ & $0.6291(2)$ & $0.0441(12)$ \\
\hline $\mathrm{H} 15$ & 0.0825 & 0.4370 & 0.6572 & $0.053^{*}$ \\
\hline C16 & $0.0924(5)$ & $0.2495(6)$ & $0.6244(3)$ & $0.0542(14)$ \\
\hline $\mathrm{H} 16$ & 0.0389 & 0.2104 & 0.6494 & $0.065^{\star}$ \\
\hline C17 & $0.1443(7)$ & $0.1731(6)$ & $0.5833(3)$ & $0.0705(19)$ \\
\hline $\mathrm{H} 17$ & 0.1245 & 0.0822 & 0.5795 & $0.085^{\star}$ \\
\hline C18 & $0.2274(7)$ & $0.2301(6)$ & $0.5466(3)$ & $0.074(2)$ \\
\hline $\mathrm{H} 18$ & 0.2642 & 0.1774 & 0.5189 & 0.089 * \\
\hline C19 & $0.2537(6)$ & $0.3653(6)$ & $0.5520(2)$ & $0.0563(15)$ \\
\hline H19 & 0.3093 & 0.4037 & 0.5279 & $0.068^{*}$ \\
\hline $\mathrm{C} 20$ & $0.2788(4)$ & $0.6858(5)$ & $0.5295(2)$ & $0.0392(11)$ \\
\hline $\mathrm{H} 20$ & 0.3492 & 0.6370 & 0.5171 & $0.047^{*}$ \\
\hline $\mathrm{C} 21$ & $0.1818(5)$ & $0.6591(7)$ & $.4836(2)$ & $0.0571(15)$ \\
\hline $\mathrm{H} 21 \mathrm{~A}$ & 0.1635 & 0.5633 & 0.4823 & 0.069 * \\
\hline $\mathrm{H} 21 \mathrm{~B}$ & 0.1107 & 0.7078 & 0.4935 & 0.069 * \\
\hline $\mathrm{C} 22$ & $0.2250(6)$ & $0.7064(9)$ & $0.4244(3)$ & $0.078(2)$ \\
\hline $\mathrm{H} 22 \mathrm{~A}$ & 0.2918 & 0.6515 & 0.4133 & $0.094^{\star}$ \\
\hline $\mathrm{H} 22 \mathrm{~B}$ & 0.1626 & 0.6936 & 0.3956 & $0.094^{\star}$ \\
\hline $\mathrm{C} 23$ & $0.2612(7)$ & $0.8536(8)$ & $0.4255(3)$ & $0.086(2)$ \\
\hline $\mathrm{H} 23 \mathrm{~A}$ & 0.2926 & 0.8780 & 0.3881 & $0.103^{*}$ \\
\hline $\mathrm{H} 23 \mathrm{~B}$ & 0.1922 & 0.9093 & 0.4320 & $0.103^{*}$ \\
\hline C24 & $0.3518(7)$ & $0.8817(7)$ & $0.4718(3)$ & $0.079(2)$ \\
\hline $\mathrm{H} 24 \mathrm{~A}$ & 0.3680 & 0.9779 & 0.4730 & $0.095^{\star}$ \\
\hline H24B & 0.4244 & 0.8354 & 0.4625 & $0.095^{\star}$ \\
\hline $\mathrm{C} 25$ & $0.3110(6)$ & $0.8352(5)$ & $0.5315(2)$ & $0.0546(15)$ \\
\hline $\mathrm{H} 25 \mathrm{~A}$ & 0.3736 & 0.8498 & 0.5601 & $0.066^{\star}$ \\
\hline $\mathrm{H} 25 \mathrm{~B}$ & 0.2430 & 0.8878 & 0.5428 & $0.066^{\star}$ \\
\hline $11^{\mathrm{a}}$ & $0.57361(4)$ & $0.64371(7)$ & $0.59102(2)$ & $0.06474(18)$ \\
\hline $03^{a}$ & $0.4217(7)$ & $0.3319(4)$ & $0.6760(3)$ & $0.0794(17)$ \\
\hline$C 7^{\mathrm{a}}$ & $0.4127(10)$ & $0.4432(3)$ & $0.6698(4)$ & $0.0794(17)$ \\
\hline$I 1 A^{b}$ & $0.4667(2)$ & $0.3832(2)$ & $0.66140(12)$ & $0.0615(6)$ \\
\hline $03 A^{b}$ & $0.5711(18)$ & $0.688(3)$ & $0.5692(9)$ & $0.0794(17)$ \\
\hline$C 7 A^{b}$ & $0.503(2)$ & $0.666(3)$ & $0.6032(10)$ & $0.0794(17)$ \\
\hline
\end{tabular}

Occupancy: $0.8,{ }^{b}$ Occupancy: 0.2 .

[Rh(hopo)(CO) $\left.\left(\mathrm{CH}_{3}\right)(\mathrm{I})\left(\mathrm{PPh}_{2} \mathrm{Cy}\right)\right]$ was prepared as follows: $(0.100 \mathrm{~g}, 0.154 \mathrm{mmol})$ was dissolved in acetone $\left(2 \mathrm{~cm}^{3}\right)$ wherafter iodomethane $(0.2186 \mathrm{~g}, 1.541 \mathrm{mmol})$ was added with stirring. The solution was stored at $25{ }^{\circ} \mathrm{C}$ for two days and yielded orange crystals (yield 68\%).

IR: $v_{\mathrm{CO}} 2056 \mathrm{~cm}^{-1} \cdot{ }^{31} \mathbf{P}$ NMR $\left(162 \mathrm{MHz}, \mathrm{CDCl}_{3}\right) \delta[\mathrm{ppm}]$ $40.96\left(\mathrm{~d},{ }^{1} J_{(\mathrm{RhP}) \mathrm{A}}=123.8 \mathrm{~Hz}\right), 37.56\left(\mathrm{~d},{ }^{1} J_{(\mathrm{RhP}) \mathrm{B}}=116.3 \mathrm{~Hz}\right)$; where $\mathrm{A}=$ major isomer, $\mathrm{B}=$ minor isomer.

\section{Experimental details}

All $\mathrm{H}$-atoms were positioned on geometrically idealized positions and refined using the riding model with fixed $\mathrm{C}-\mathrm{H}$ distances for aromatic $\mathrm{C}-\mathrm{H}$ of $0.93 \AA(\mathrm{C}-\mathrm{H})\left[U_{\text {iso }}(\mathrm{H})=1.2\right.$ $\left.U_{\text {eq }}\right]$, for methyl C $-\mathrm{H}$ of $0.96 \AA(\mathrm{C}-\mathrm{H})\left[U_{\text {iso }}(\mathrm{H})=1.5 U_{\text {eq }}\right]$, for methylene $\mathrm{C}-\mathrm{H}$ of $0.97 \AA(\mathrm{C}-\mathrm{H})\left[U_{\text {iso }}(\mathrm{H})=1.5 U_{\text {eq }}\right]$ and for methine $\mathrm{C}-\mathrm{H}$ of $0.98 \AA$ ( $\mathrm{C}-\mathrm{H})\left[U_{\text {iso }}(\mathrm{H})=1.5 U_{\text {eq }}\right]$. The graphics were obtained using the DIAMOND [3] program with 50\% probability ellipsoids. The highest residual peak is located $0.77 \AA$ from $\mathrm{Rh}$ and the deepest hole is situated $0.71 \AA$ from $\mathrm{Rh}$. The $\mathrm{I} 1$ and $\mathrm{C7}-\mathrm{O} 3$ (carbonyl group) atoms are disordered in an approximate $80 \%$ ratio with the higher value attributed to the I1 trans methyl and the $20 \%$ isomer and hydrogen atoms in the figure are omitted for clarity.

\section{Comment}

The iodomethane oxidative addition reaction to $\mathrm{Rh}$ (I) complexes to form alkyl and acyl product is an essential and relevant reaction in catalytic processes as utilized in industry to produce acetic acid from methanol and carbon monoxide in the Monsanto process [5-7]. It is known that only one CO ligand in the precursor $\left[\mathrm{Rh}(\mathrm{BID})(\mathrm{CO})_{2}\right]$ complexes [where BID denote different monocharged bidentate ligands such as cupferrate and 2-oxopyridine $N$-oxide, etc.] can be substituted by tertiary phosphine ligands $\left(\mathrm{PX}_{3}\right)$ to form $\left[\mathrm{Rh}(\mathrm{BID})(\mathrm{CO})\left(\mathrm{PX}_{3}\right)\right]$ complexes. These complexes have been intensively studied as possible catalysts in model catalytic reactions [8-16].

The title complex displays a distorted octahedral geometry around the $\mathrm{Rh}$ centre indicated by the small bite angle of $79.42(1)^{\circ}$ of the five membered chelate ring as well as the $\mathrm{C} 7-$ $\mathrm{Rh}-\mathrm{C} 6, \mathrm{C} 6-\mathrm{Rh}-\mathrm{P} 1, \mathrm{P} 1-\mathrm{Rh}-\mathrm{I} 1, \mathrm{P} 1-\mathrm{Rh}-\mathrm{O} 2$ and $\mathrm{I} 1-\mathrm{Rh}-\mathrm{C} 6$ angles of 87.24(1), 91.19(8), 96.58(1), 174.32(1) and 171.08(1) ${ }^{\circ}$, respectively. The coordination mode in the title complex is similar to the complex reported previously [11, 17]. The $\mathrm{Rh}-01, \mathrm{Rh}-02, \mathrm{Rh}-\mathrm{P} 1, \mathrm{Rh}-\mathrm{C} 6$ and $\mathrm{Rh}-\mathrm{I} 1$ bond distances are 2.0494(1), 2.1108(5), 2.2926(6), 2.0930(5) and 2.7448(7) , respectively. The lengthening of $\mathrm{Rh}-\mathrm{O} 2$ compared to $\mathrm{Rh}-\mathrm{O} 1$ is due to the strong trans influence of the $\mathrm{P}$ atom. In the figure the hydrogen atoms have been omitted for clarity.

Acknowledgements: The authors would like to thank the University of the Free State for financial support.

\section{References}

1. Sheldrick, G. M.: A short history of SHELX. Acta Crystallogr. A64 (2008) 112-122.

2. Bruker. SAINT-Plus (Version 7.12) and SADABS (Version 2004/1). Bruker AXS Inc., Madison, WI, USA (2004).

3. Brandenburg, K.: DIAMOND. Visual Crystal Structure Information System. 3.0c. Crystal Impact, Bonn, Germany (2005).

4. Dolomanov, O. V.; Bourhis, L. J.; Gildea, R. J.; Howard, J. A. K.; Puschmann, H.: OLEX2: A complete structure solution, refinement and analysis program. J. Appl. Crystallogr. 42 (2009) 339-341.

5. Elmakki, M. A.; Alexander, O. T.; Venter, G. J. S.; Venter, J. A.: Crystal structure of carbonyl(2-oxopyridin- $1\left(2 \mathrm{H}\right.$-olato- $\left.\mathrm{K}^{2} \mathrm{O}, \mathrm{O}^{\prime}\right)$ 
(diphenylcyclohexylphosphine- $\mathrm{K} P$ ) rhodium(I), $\mathrm{C}_{24} \mathrm{H}_{25} \mathrm{NO}_{3} \mathrm{PRh}$. Z. Kristallogr. NCS 232 (2017) 831-833.

6. Venter, J. A.; Leipoldt, J. G.; Eldik, R. V.: Solvent, temperature, and pressure dependence of oxidative addition of iodomethane to complexes of the type $\mathrm{Rh}^{\prime}(\beta$-diketone) (CO) $\left(\mathrm{PPh}_{3}\right)$. Inorg. Chem. 30 (1991) 2207-2209.

7. Hallinan, N.; Hinnenkamp, J.: Rhodium-catalyzed methanol carbonylation: new low water technology. Chem. Ind. 82 (2001) 545.

8. Basson, S. S.; Leipoldt, J. G.; Roodt, A.; Venter, J. A.: Mechanism for the oxidative addition of iodomethane to carbonyl( $\mathrm{N}$-hydroxy- $\mathrm{N}$-nitrosobenzenaminato- $\left.\mathrm{K}^{2} \mathrm{O}-\mathrm{O}^{\prime}\right)$ triarylphosphinerhodium(I) complexes and crystal structure of $\left[\mathrm{Rh}\right.$ (cupf)(CO) $\left.\left(\mathrm{CH}_{3}\right)(\mathrm{I})\left(\mathrm{PPh}_{3}\right)\right]$. Inorg. Chim. Acta 128 (1987) 31-37.

9. Brink, A.; Roodt, A.; Steyl, G.; Visser, H. G.: Steric vs. electronic anomaly observed from iodomethane oxidative addition to tertiary phosphine modified rhodium(I) acetylacetonato complexes following progressive phenyl replacement by cyclohexyl $\left[\mathrm{PR}_{3}=\mathrm{PPh}_{3}, \mathrm{PPh}_{2} \mathrm{Cy}, \mathrm{PPhCy}_{2}, \mathrm{PCy}_{3}\right]$. Dalton Trans. 39 (2010) 5572-5578.

10. Elmakki, M. A.; Koen, R.; Venter, J. A.; Drost, R.: Crystal structure of dicarbonyl(pyridine-2-olate-1-oxido- $\mathrm{K}^{2} \mathrm{O}, \mathrm{O}^{\prime}$ )rhodium(I), $\mathrm{C}_{7} \mathrm{H}_{4} \mathrm{NO}_{4}$ Rh. Z. Kristallogr. NCS 231 (2016) 703-705.

11. Elmakki, M. A.; Koen, R.; Drost, R. M.; Alexander, O. T.; Venter, G. J. S.; Venter, J. A.: Crystal structure of carbonyl (2-oxopyridin-1(2H-olato- $\left.\mathrm{K}^{2} \mathrm{O}, \mathrm{O}^{\prime}\right)$ (triphenylphosphine- $\mathrm{K} P$ )
rhodium(I), $\mathrm{C}_{24} \mathrm{H}_{19} \mathrm{NO}_{3}$ PRh. Z. Kristallogr. NCS 231 (2016) 781-783.

12. Roodt, A.; Visser, H. G.; Brink, A.: Structure/reactivity relationships and mechanism from X-ray data and spectroscopic kinetic analysis. Crystallogr. Rev. 17 (2011) 241-280.

13. Roodt, A.; Otto, S.; Steyl, G.: Structure \& solution behaviour of rhodium(I) Vaska-type complexes for correlation of steric \& electronic properties of tertiary phosphine ligands. Coord. Chem. Rev. 245 (2003) 121-137.

14. Warsink, S.; Kotze, P. D. R.; Janse van Rensburg, J. M.; Venter, J. A.; Otto, S.; Botha, E.; Roodt, A.: Kinetic-mechanistic and solid-state study of the oxidative addition and migratory insertion of iodomethane to [Rhodium(S,O-BdiPT or N,Oox)(CO) $\left.\left(P R^{1} R^{2} R^{3}\right)\right]$ complexes. Eur. J. Inorg. Chem. 32 (2018) 3615-3625.

15. Conradie, M. M.; van Rooyen, P. H.; Pretorius, C.; Roodt, A.; Conradie, J.: Rhodium-rhodium interactions in $[\mathrm{Rh}(\beta-$ diketonato)(CO $)_{2}$ ] complexes. J. Mol. Struct. 1144 (2017) 280-289.

16. Dobrynin, M. V.; Pretorius, C.; Kama, D. V.; Roodt, A.; Boyarskiy, V. P.; Islamova, R. M.: Rhodium(I)-catalysed crosslinking of polysiloxanes conducted at room temperature. J. Catal. 372 (2019) 193-200.

17. Elmakki, M. A.; Renier, K.; Venter, J. A.; Drost, R.: Crystal structure of dicarbonyl(pyridin-2-olate-1-oxido-k2O, $\mathrm{O}^{\prime}$ ) rhodium(I), $\mathrm{C}_{7} \mathrm{H}_{4} \mathrm{NO}_{4}$ Rh. Z. Kristallogr. NCS 231 (2016) 703-705. 